Relations industrielles

Industrial Relations

\title{
Cadres : la cote d'alerte, par Emmanuel Kay, Paris, Centor, Entreprises Moderne d'Édition, 1975, 156 pp.
}

\section{Laurent Bélanger}

Volume 31, numéro 2, 1976

URI : https://id.erudit.org/iderudit/028711ar

DOI : https://doi.org/10.7202/028711ar

Aller au sommaire du numéro

Éditeur(s)

Département des relations industrielles de l'Université Laval

ISSN

0034-379X (imprimé)

1703-8138 (numérique)

Découvrir la revue

Citer ce compte rendu

Bélanger, L. (1976). Compte rendu de [Cadres : la cote d'alerte, par Emmanuel Kay, Paris, Centor, Entreprises Moderne d'Édition, 1975, 156 pp.] Relations industrielles / Industrial Relations, 31(2), 322-322.

https://doi.org/10.7202/028711ar

Tous droits réservés @ Département des relations industrielles de l'Université Laval, 1976
Ce document est protégé par la loi sur le droit d'auteur. L'utilisation des services d'Érudit (y compris la reproduction) est assujettie à sa politique d'utilisation que vous pouvez consulter en ligne.

https://apropos.erudit.org/fr/usagers/politique-dutilisation/ 


\section{RECENSIONS BOOK REVIEWS}

Cadres: la cote d'alerte, par Emmanuel Kay, Paris, Centor, Entreprises Moderne d'Édition, 1975, 156 pp.

C'est un ouvrage publié d'abord en langue anglaise par l'American Management Association, en 1974, sous le titre The Crisis in Middle Management. L'auteur va beaucoup plus loin qu'une simple description du malaise des cadres ou de la crise des quarante ans. En se basant sur sa propre expérience de consultant et en rapaillant les quelques données empiriques disponibles sur le sujet, l'auteur aborde la manière dont les cadres réagissent individuellement à cette crise et discute des facteurs qui peuvent attirer les cadres vers la syndicalisation ou les en éloigner. Sans tomber dans une panoplie de recettes pour contrer ce malaise, l'auteur fait état d'un éventail de possibilités de progrès individuel et d'avancement qui pourraient être offertes aux cadres intermédiaires, si les directions générales voulaient sortir des sentiers battus pour concevoir et initier des changements importants au plan des structures et des processus de gestion.

En initiant un programme de développement de l'organisation (D.O.), en introduisant un nouveau système de management tel que la D.P.O., en procédant à une restructuration horizontale et verticale des tâches, en améliorant les systèmes d'appréciation du personnel et en faisant une meilleure utilisation de la structure matricielle, la haute-direction créerait ainsi un ensemble de conditions qui rendraient les organisations plus efficaces et qui amélioreraient le climat ou le milieu organisationnel, conditions qui m'apparaissent nécessaires pour enrayer l'apathie ou le désintéressement des cadres intermédiaires et de premier palier.

On connaît l'ensemble des difficultés qu'on éprouve avec les systèmes actuels d'appréciation du personnel. L'auteur, en plus de relever ces difficultés, se donnent la peine d'esquisser ce que devraient être les systèmes d'appréciation. Au lieu de poursuivre une multitude d'objectifs avec un système qui prévaut à l'échelle de l'en- treprise pour les diverses catégories de personnel, il serait plus approprié de mettre sur pied autant de systèmes d'appréciation qu'il existe d'objectifs. Ce nouveau point de départ permet à l'auteur de décrire les caractéristiques et le fonctionnement de chacun de ces systèmes. En poursuivant ainsi une réflexion critique sur les pratiques managériales actuelles, l'auteur en arrive dans ses deux derniers chapitres à établir un profil du rôle des cadres dans l'entreprise de demain et à préciser les initiatives que les cadres et les directions générales devront prendre dans l'avenir.

En plus de décrire la situation que vivent actuellement les cadres, l'ouvrage se veut donc d'un intérêt pratique par les orientations concrètes qu'il propose et qu'il appuie sur des expériences déjà réalisées dans quelques entreprises.

Université Laval

\section{Laurent BÉLANGER}

Le développement des organisationis : sa pratique, ses perspectives et ses problèmes, par Warren G. Bennis, Paris, Dalloz, Coll. Gestion, 1975, 100 pp.

Le développement des organisations, stratégies et modèles, par Richard Beckard, Paris, Dalloz, Coll. Gestion, 1975, 143 pp.

La maison Dalloz mérite certainement des félicitations pour sa collection «Gestion», qui nous présente la traduction de deux ouvrages fondamentaux en matière de développement des organisations. Ces volumes de Bennis et Beckard font partie d'une collection de six publiée par la maison Addison Wesley en 1969. Déjà largement connus et diffusés en milieu anglophone, ces volumes de base sont maintenant accessibles au lecteur francophone, et cela, dans une traduction soignée, utilisant des termes à la portée de tous ceux qui s'intéressent au domaine de l'application des sciences du comportement.

Même si les titres se ressemblent, les deux ouvrages diffèrent quant à la présentation et au fond. Bennis essaie de re- 